\title{
Levodopa-carbidopa intestinal gel in a subgroup of patients with dyskinesia at baseline from the GLORIA Registry
}

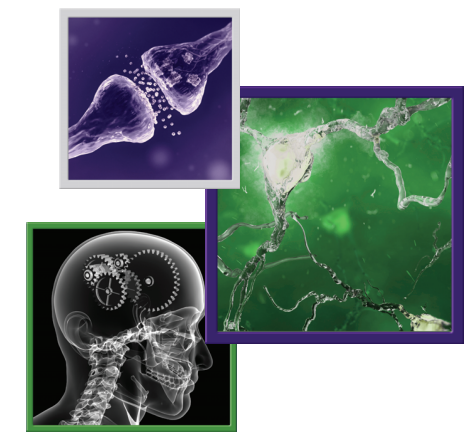

\author{
Werner Poewe ${ }^{1}$, K Ray Chaudhuri², Lars Bergmann ${ }^{3}$ \& Angelo Antonini*,4 \\ ${ }^{1}$ Department of Neurology, Medical University of Innsbruck, Innsbruck, Austria \\ ${ }^{2}$ Institute of Psychiatry, Psychology \& Neuroscience at King's College London \& King's College Hospital (KCH) NHS Foundation \\ Trust, London, UK \\ ${ }^{3}$ AbbVie Inc., North Chicago, IL, USA \\ ${ }^{4}$ Department of Neuroscience, Padua University, Padua, Italy \\ *Author for correspondence: Tel.: +39 041220 7554; angelo3000@yahoo.com
}

\section{Practice points}

- Prolonged levodopa use in advanced Parkinson's disease patients is associated with motor complications including motor response fluctuations and levodopa-induced dyskinesia.

- This was a post hoc analysis of GLORIA, a 24-month observational registry that evaluated long-term effectiveness of levodopa-carbidopa intestinal gel (LCIG) in a real-world setting.

- Patients were divided into subgroups based on self-reported $<4$ (low) and $\geq 4$ (high) h/day of baseline dyskinesia.

- Mean duration of dyskinesia improved in patients with high dyskinesia burden at baseline and increased in patients with low dyskinesia burden at baseline.

- At the same time, dyskinesia severity and pain associated with dyskinesia improved to a greater extent in patients with high versus low baseline dyskinesia, with numerical improvements in the low dyskinesia burden group.

- LCIG treatment markedly improved quality of life in patients with low and high baseline dyskinesia.

- Adverse drug reactions occurred at a similar rate in both subgroups.

- Despite increases in levodopa dose, LCIG treatment led to significant and sustained reductions in dyskinesia time, severity and associated pain in advanced Parkinson's disease patients and high dyskinesia burden at baseline.

Aim: To evaluate long-term effects of levodopa-carbidopa intestinal gel on dyskinesia burden. Patients \& methods: Post hoc analysis of the GLORIA registry assessed subgroups of advanced Parkinson's disease patients with $<4$ and $\geq 4 \mathrm{~h}$ /day of levodopa-induced dyskinesia at baseline. Results \& conclusions: Mean dyskinesia duration significantly $(p<0.0001)$ decreased by $3.5 \mathrm{~h}$ in patients with $\geq 4 \mathrm{~h}$ baseline dyskinesia; conversely, dyskinesia duration increased by $1.6 \mathrm{~h}$ in patients with $<4 \mathrm{~h}$ baseline dyskinesia. Quality of life improved in both subgroups. Adverse drug reactions occurred at similar rates in both subgroups. Despite increases in levodopa dose, levodopa-carbidopa intestinal gel treatment led to significant and sustained reductions in dyskinesia time, severity and associated pain in advanced Parkinson's disease patients with high baseline dyskinesia burden.

First draft submitted: 31 August 2018; Accepted for publication: 6 November 2018; Published online: 14 December 2018

Keywords: dyskinesia $\bullet$ levodopa $\bullet$ Parkinson's disease $\bullet$ quality of life

Levodopa is the most effective treatment for Parkinson's disease (PD), but prolonged use in patients is associated with motor complications including motor and nonmotor response fluctuations and a range of levodopa-induced dyskinesias [1-4]. Motor complications are thought to be caused by fluctuating levodopa blood levels due to irregular gastric emptying and intestinal levodopa absorption (delayed 'On' and no 'On'), the short half-life of levodopa and resulting maladaptive neuroplasticity [5,6]. Motor and nonmotor complications have been shown to negatively impact quality of life (QoL) in patients with advanced PD.

Levodopa-carbidopa intestinal gel (LCIG, designated in the USA as carbidopa-levodopa enteral suspension [CLES]; Duopa ${ }^{\circledR} /$ Duodopa ${ }^{\circledR}$, AbbVie Inc., IL, USA) is delivered continuously via percutaneous endoscopic gastrostomy with a jejunal extension tube (PEG-J) and is a long-term treatment option for motor fluctuations in 
patients with advanced PD who are refractory to oral medication. LCIG has been shown to reduce motor and nonmotor complications and improve QoL in patients with advanced PD [7-10].

The GLORIA registry [11,12] evaluated the long-term efficacy and safety of LCIG in routine care of patients with advanced PD. This post hoc analysis of the GLORIA registry evaluated the long-term effect of LCIG on dyskinesia burden (dyskinesia duration and severity) in subgroups of patients.

\section{Methods}

Study design \& treatment

GLORIA is a multinational observational registry designed to collect efficacy and safety data of routine clinical care use of LCIG over 24 months. This registry has been described previously in more detail [11,12]. GLORIA was conducted according to Good Clinical Practice Guidelines and Institutional Review Board requirements, as applicable.

Patients were individually titrated to LCIG via nasojejunal (NJ) tube. After PEG-J placement, patients were treated for up to 24 months with LCIG. Concomitant PD medications were allowed at the discretion of the investigator.

\section{Patients}

Patients were enrolled in the registry according to the European Summary of Product Characteristics (having advanced levodopa-responsive PD with persistent severe motor fluctuations with or without troublesome dyskinesia) and national reimbursement criteria, where applicable. Patients were either LCIG naive or had received LCIG for a maximum of 12 months before enrollment. Observations were prospective for LCIG-naive patients and were partially retrospective and prospective for patients with previous LCIG experience.

\section{Assessments}

Efficacy assessment

Efficacy outcomes described in this analysis include the mean change from baseline to study visit as evaluated by the Unified Parkinson's Disease Rating Scale (UPDRS) Part IV (complications of therapy including dyskinesia); items 32 and 39 were modified using the rating instructions for the corresponding parts 4.3 and 4.1 of the Movement Disorder Society (MDS)-UPDRS to allow for collection of actual hours of 'Off' time and 'On' time with dyskinesia as reported by the patient for the week prior to the clinical visit. UPDRS Part II (activities of daily living) and Part III (motor examination) were also assessed (in the 'On' state). Patient QoL was assessed via the 8-item Parkinson's Disease Questionnaire (PDQ-8). Efficacy was assessed at baseline before the initiation of LCIG via NJ tube; at discharge from hospital following PEG-J placement (day 1); and at 6, 12, 18 and 24 months after PEG-J placement.

\section{Safety assessment}

Adverse drug reactions (ADRs) included all adverse events that had a reasonable possibility of being related to the treatment drug or device (as determined by the investigator). ADRs were recorded for the duration of the registry study and for 28 days after the patient's last visit.

\section{Statistical analysis}

For this posthoc analysis, patients were allocated into two subgroups based on baseline hours/day of dyskinesia defined as low $(<4 \mathrm{~h} /$ day) and high $(\geq 4 \mathrm{~h} /$ day). Patients without baseline dyskinesia assessment were excluded from the analysis. Statistical significance in the change from baseline to each time point for each efficacy measure was evaluated using a paired $t$-test.

\section{Results}

Patients

Out of 375 patients in the GLORIA registry, there were 118 patients (31\%) with $<4 \mathrm{~h} /$ day of dyskinesia at baseline and 139 patients (37\%) with $\geq 4 \mathrm{~h} /$ day dyskinesia at baseline (118 patients [31\%] did not have measurements of dyskinesia duration at baseline). Overall, 258 patients (69\%) completed the registry; cumulative withdrawals were higher in patients with high versus low baseline dyskinesia (51 [37\%] vs 36 [31\%] patients). The most prominent reasons for withdrawal were ADRs $(n=46 ; 12.3 \%)$, withdrawal of informed consent $(n=31 ; 8.3 \%)$, patients lost to follow-up $(\mathrm{n}=16 ; 4.3 \%)$ and lack of efficacy $(\mathrm{n}=11 ; 2.9 \%)$. Demographics were similar across the subgroups 
Table 1. Baseline demographics and characteristics.

\begin{tabular}{|c|c|c|}
\hline \multirow[t]{2}{*}{ Characteristic } & \multicolumn{2}{|c|}{ Baseline dyskinesia subgroup } \\
\hline & $<4 \mathrm{~h} /$ day $(n=118)$ & $\geq 4$ h $/$ day $(n=139)$ \\
\hline Age, mean (SD) & $66.4(8.1)$ & $64.9(9.7)$ \\
\hline \multicolumn{3}{|l|}{ Sex, $n(\%):$} \\
\hline - Female & $41(35)$ & $71(51)$ \\
\hline - Male & $77(65)$ & $68(49)$ \\
\hline Years since PD diagnosis, mean (SD) & $11.9(6.5)$ & $13.3(6.4)$ \\
\hline Total daily levodopa dose (mg), mean (SD) & $998.7(450.1)$ & $859.7(447.4)$ \\
\hline $\begin{array}{l}\text { Hours/day of 'Off' time (UPDRS IV: modified item 39), } \\
\text { mean (SD) }\end{array}$ & $6.4(3.5)^{\dagger}$ & $5.8(2.9)^{\dagger}$ \\
\hline $\begin{array}{l}\text { Hours/day of dyskinesia (UPDRS IV: modified item 32), } \\
\text { mean (SD) }\end{array}$ & $1.0(1.2)^{\dagger}$ & $7.1(2.8)^{\dagger}$ \\
\hline Activities of daily living (UPDRS II) 'On', mean (SD) & $16.6(8.9)^{\dagger}$ & $16.3(10.9)^{\dagger}$ \\
\hline Motor examination (UPDRS III) 'On', mean (SD) & $25.0(12.5)^{\dagger}$ & $23.4(11.6)^{\dagger}$ \\
\hline Quality of life (PDQ-8), mean (SD) & $44.6(19.1)^{\dagger}$ & $48.4(19.2)^{\dagger}$ \\
\hline
\end{tabular}

Table 2. Levodopa-equivalent doses by treatment group and visit.

\begin{tabular}{|c|c|c|c|c|c|c|c|c|}
\hline Visit & \multicolumn{4}{|c|}{$<4 \mathrm{~h} /$ day } & \multicolumn{4}{|c|}{$\geq 4 \mathrm{~h} /$ day } \\
\hline Day 1 & 30 & $1658.3(678.4)$ & 77 & $2191.5(846.1)$ & 58 & $1381.3(651.4)$ & 70 & $1787.1(793.8)$ \\
\hline Month 12 & 32 & $1945.2(721.1)$ & 61 & $2223.5(837.8)$ & 49 & $1674.0(874.4)$ & 57 & $1776.5(829.3)$ \\
\hline Month 18 & 22 & $1895.6(744.0)$ & 63 & $2119.7(805.9)$ & 46 & $1669.9(884.7)$ & 50 & $1841.9(737.1)$ \\
\hline
\end{tabular}

(Table 1). Patients with $<4 \mathrm{~h} /$ day of dyskinesia at baseline had slightly shorter PD duration, a slightly higher total daily levodopa dose, a slightly higher daily 'Off' time and fewer hours per day of dyskinesia. Activities of daily living, motor symptoms and QoL were similar in both subgroups.

During 24 months of treatment, patients on LCIG monotherapy (e.g., no adjunctive therapy during the $16 \mathrm{~h}$ of LCIG infusion) had similar increases in levodopa-equivalent doses (LEDs), regardless of baseline dyskinesia (Table 2). The LEDs for patients on LCIG polytherapy (LCIG and $\geq 1$ other anti-PD drug) were generally stable over 24 months with LEDs starting and ending at $2191.5(846.1) \mathrm{mg} / \mathrm{day}$ and $2214.5(840.3) \mathrm{mg} /$ day, respectively, for patients with $<4 \mathrm{~h} /$ day baseline dyskinesia, and starting and ending at 1787.1 (793.8) $\mathrm{mg} / \mathrm{day}$ and 1787.6 (769.7) $\mathrm{mg} /$ day, respectively, for patients with $\geq 4 \mathrm{~h} /$ day baseline dyskinesia.

\section{Efficacy}

'Off'time

Mean 'Off' time significantly decreased and remained stable for the study duration in both high and low baseline dyskinesia subgroups ( $\mathrm{p}<0.0001$ for both groups) (Figure 1A). By month 24, the mean (standard deviation [SD]) 'Off' time reduced by 3.9 (3.4) h in patients with high baseline dyskinesia and reduced by 4.4 (3.6) h in patients with low baseline dyskinesia ( $\mathrm{p}<0.0001$ for both).

\section{Dyskinesia}

In patients with high baseline dyskinesia, the mean (SD) duration of dyskinesia decreased and remained stable through month 24 (reduction of 3.5 [4.6] h; p $<0.0001$; Figure 1B). In patients with low baseline dyskinesia, however, the mean duration of dyskinesia increased and maintained that increase through 24 months of treatment $(+1.6[3.3] \mathrm{h} ; \mathrm{p}<0.0001)$. 
(A)

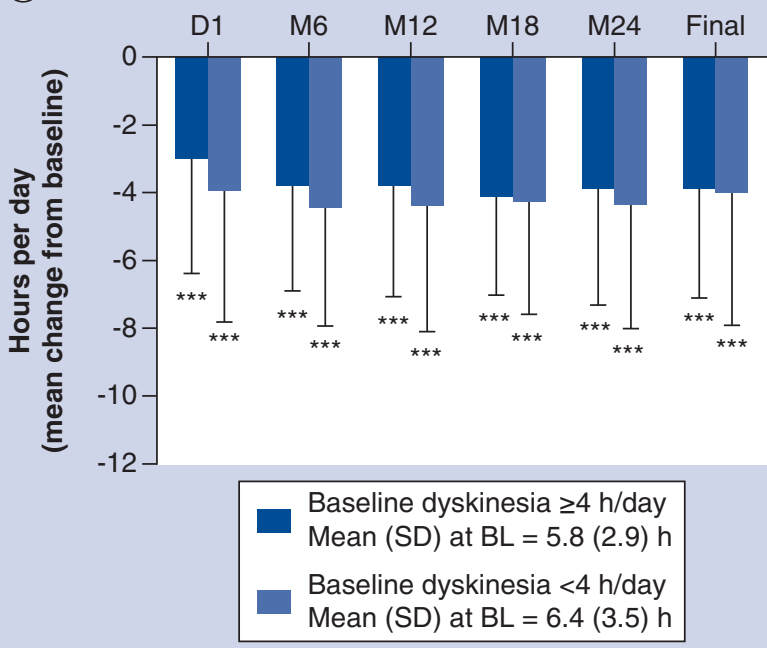

(C)

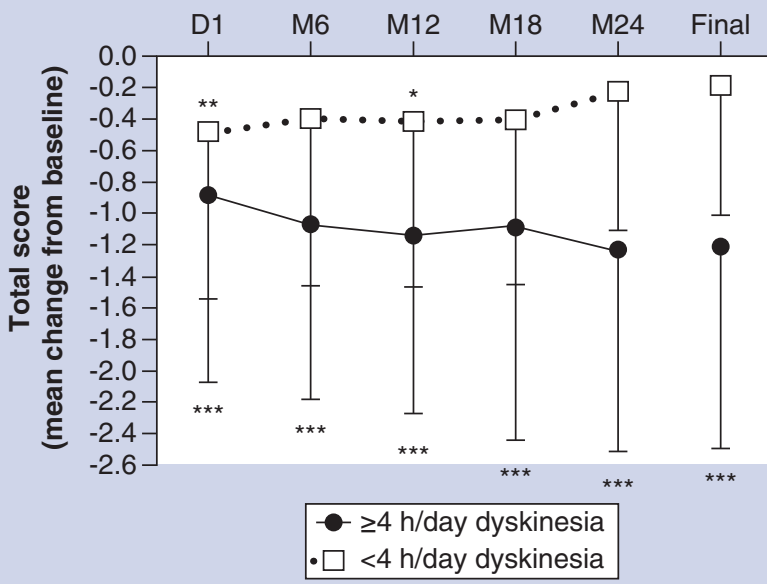

(E)

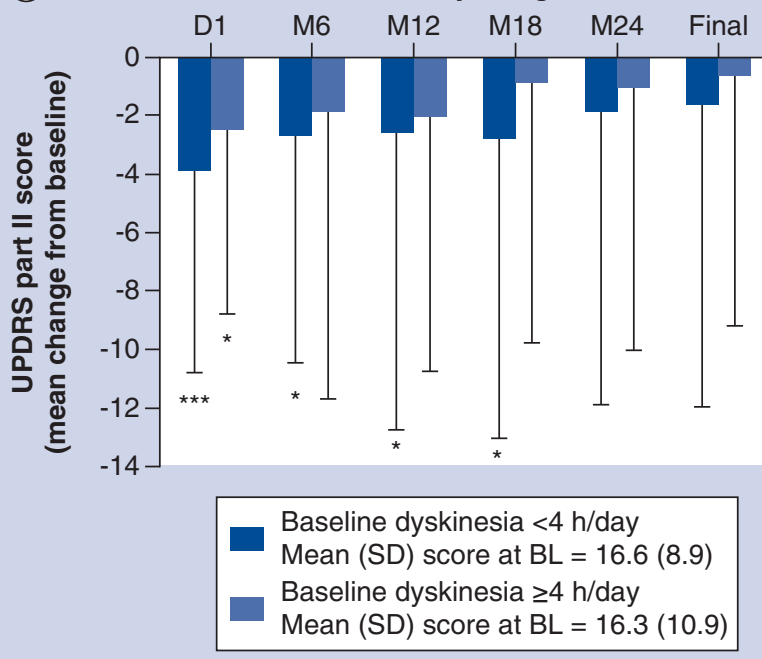

(B) Dyskinesia: UPDRS IV (modified item 32)

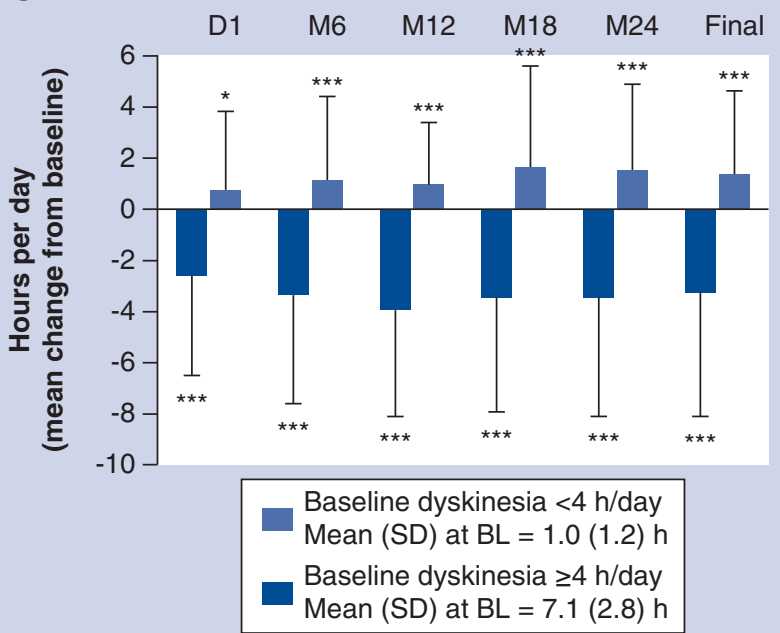

(D)

\section{UPDRS IV item 34 (pain)}

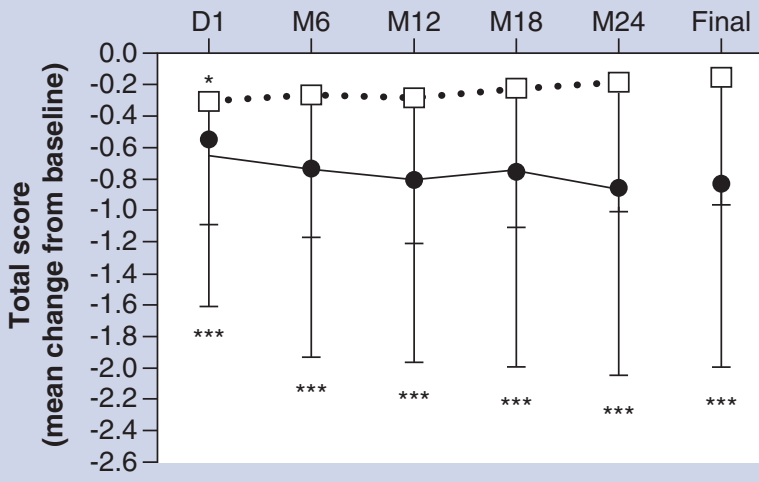

(F) $\cdot \square$ h/day dyskinesia
$\cdot \square \cdot<4$ h/day dyskinesia

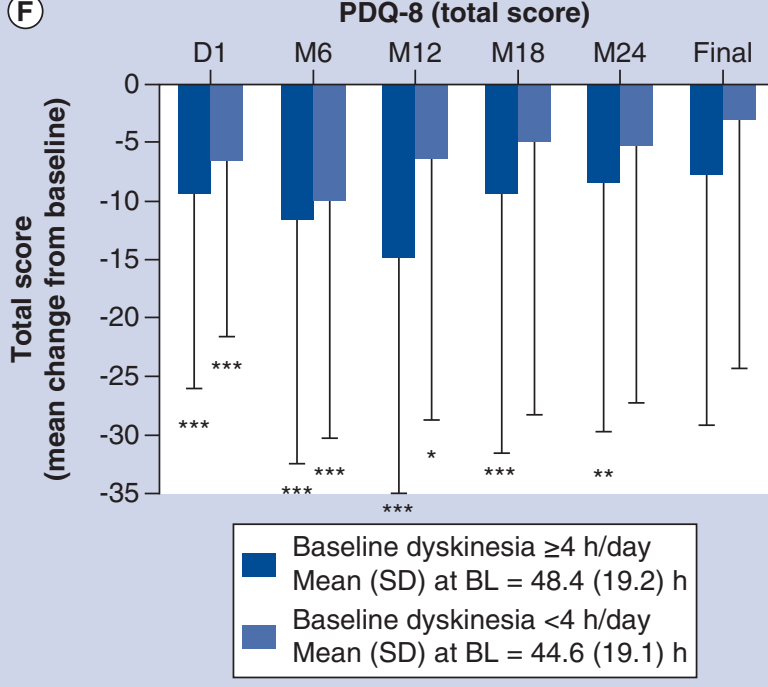

Figure 1. Motor, nonmotor, and quality-of-life outcomes in patients with $\geq 4$ and $<4 \mathrm{~h}$ /day baseline dyskinesia after 24 months of LCIG treatment. Statistics indicate improvement from baseline. ${ }^{*} p<0.05,{ }^{*} p<0.01,{ }^{*} * p<0.001$.

BL: Baseline; LCIG: Levodopa-carbidopa intestinal gel; PDQ 8: 8-item Parkinson's Disease Questionnaire; SD: Standard deviation; UPDRS: Unified Parkinson's Disease Rating Scale. 
Table 3. Most common nondevice-related ( $\geq 3 \%$ in either group) adverse drug reactions.

\begin{tabular}{|c|c|c|}
\hline Summary, $\mathrm{n}(\%)$ & & inesia subgroup \\
\hline & $<4 \mathrm{~h} /$ day $(n=110)$ & $\geq 4 \mathrm{~h} /$ day $(n=117)$ \\
\hline Any ADR & $56(50.9)$ & $65(50.0)$ \\
\hline Serious & $31(28.2)$ & $29(22.3)$ \\
\hline Discontinuation due to ADR & $5(4.5)$ & $12(9.2)$ \\
\hline Preferred term, n (\%) & $<4$ h/day $(n=110)$ & $\geq 4 \mathrm{~h} /$ day $(n=130)$ \\
\hline Hallucination & $6(5.5)$ & $2(1.5)$ \\
\hline Abdominal pain & $6(5.5)$ & $2(1.5)$ \\
\hline Dyskinesia & $3(2.7)$ & $4(3.1)$ \\
\hline Parkinsonism & $2(1.8)$ & $5(3.8)$ \\
\hline Psychotic disorder & $1(0.9)$ & $4(3.1)$ \\
\hline
\end{tabular}

Dyskinesia severity (UPDRS item 33) improved significantly over 24 months of treatment in patients with $\geq 4$ h baseline dyskinesia ( $p<0.0001$ for all time points; Figure $1 \mathrm{C}$ ). In patients with low baseline dyskinesia, the severity of dyskinesia numerically improved with statistical significance at month 12 ( $\mathrm{p}<0.05$ ). Pain associated with dyskinesia (UPDRS item 34) also improved numerically with statistical significance at day $1(\mathrm{p}<0.05)$ in patients who had low and high baseline dyskinesia, but was only reduced in patients who had high baseline dyskinesia duration through month 24 ( $p<0.0001$ for all time points) (Figure 1D).

\section{Other motor symptoms}

Motor scores (UPDRS Part III) decreased from baseline, regardless of baseline dyskinesia. More specifically, after initiation of LCIG, patients experienced significant $(\mathrm{p}<0.0001)$ reductions from baseline in UPDRS Part III mean (SD) scores of -4.3 (8.7) and -4.5 (7.4) in patients with $<4 \mathrm{~h} /$ day and $\geq 4 \mathrm{~h} /$ day baseline dyskinesia, respectively. After 24 months, patients with low and high baseline dyskinesia had reductions from baseline in UPDRS Part III mean scores of $-1.6(10.8)$ and -2.9 (12.8), respectively, although these reductions were not statistically significant.

\section{Activities of daily living \& QoL}

The ability to perform activities of daily living, as measured by UPDRS Part II, improved in both patient subgroups (Figure 1E). Through month 18, patients with high baseline dyskinesia experienced significant ( $\mathrm{p}<$ 0.05 ) improvements from baseline; by month 24 , scores were still numerically, but not significantly, reduced from baseline in both subgroups.

LCIG treatment led to significant improvements in QoL in both dyskinesia subgroups, although improvements were greater in patients with high baseline dyskinesia (Figure 1F). After LCIG initiation, PDQ-8 scores were significantly ( $p<0.05$ for all) reduced from baseline until month 12 for patients with low baseline dyskinesia and until month 24 ( $\mathrm{p}<0.01$ for all) for patients with high baseline dyskinesia.

\section{Safety}

Overall, the proportion of patients experiencing ADRs was similar in both the high and low baseline dyskinesia subgroups (Table 3). Serious ADRs occurred slightly more frequently in the low versus high baseline dyskinesia duration subgroup ( 28.2 vs $22.3 \%$, respectively), although fewer patients with low baseline dyskinesia experienced severe ADRs (12.7 vs 16.9\%, respectively). The most common ADR leading to LCIG discontinuation that occurred in two patients was device dislocation; all other ADRs leading to discontinuation occurred in one patient each. ADRs leading to LCIG discontinuation were reported by more patients with high baseline dyskinesia than by patients with low baseline dyskinesia. The most commonly reported nondevice-related ADRs (occurring in $\geq 3 \%$ 
of patients in either group) were decreased weight, polyneuropathy, Parkinsonism, dyskinesia, psychotic disorder, depression, abdominal pain, hallucination and fall.

\section{Discussion}

In this posthoc analysis of the GLORIA study, LCIG-treated patients with advanced PD who had $\geq 4 \mathrm{~h} /$ day of dyskinesia at baseline had significant and sustained reductions in dyskinesia time, severity and associated pain over 24 months of treatment, despite an increase in levodopa-equivalent daily dose. These results align with the concept that changing the pattern of levodopa delivery from pulsatile to continuous is a critical factor in reducing pre-existing levodopa-induced dyskinesias [13-16]. Previous studies with continuous drug delivery schemes, both of levodopa with LCIG and apomorphine (subcutaneous), have likewise shown reductions in dyskinesia burden despite increases in LED [17-19]. Imaging data using raclopride positron emission tomography scan have indeed suggested sustained synaptic dopamine release with continuous intrajejunal levodopa infusion [20]. These data expand on previous reports of the effect of LCIG on dyskinesia in patients with advanced PD [18,19], with a larger patient population observed over a longer period of time in the current study.

Patients with $<4 \mathrm{~h} /$ day of dyskinesia at baseline experienced increased duration of dyskinesia over the course of the study, but at the same time there was numerical improvement of dyskinesia severity. Pain associated with dyskinesia and QoL also improved indicating that increased dyskinesia duration was due to nontroublesome involuntary movements.

As expected, LCIG treatment led to a significant decrease in 'Off' time, regardless of baseline dyskinesia duration. Patients with $\geq 4 \mathrm{~h}$ /day of dyskinesia at baseline experienced significant improvements in QoL over 24 months of LCIG treatment; those with low dyskinesia burden at baseline did so through month 12. ADRs were consistent with the known safety profile of LCIG and were comparable between subgroups.

Although this open-label, noncontrolled registry is limited also by its posthoc nature, it provides a clearer understanding of the impact of continuous administration of levodopa on dyskinesia, which has not been extensively studied. Future prospective studies of the effect of LCIG on dyskinesia in patients with advanced PD are warranted. One such study (NCT02799381) is ongoing and is using the Unified Dyskinesia Rating Scale with the primary outcome being the effect of LCIG on dyskinesia in advanced PD patients.

Open access

This work is licensed under the Attribution-NonCommercial-NoDerivatives 4.0 Unported License. To view a copy of this license, visit http://creativecommons.org/licenses/by-nc-nd/4.0/

\section{Author contributions}

All authors were involved in the concept/design of the study, data interpretation, review and critique of the manuscript throughout the editorial process and approval of the final manuscript draft for publication. In addition, W Poewe and KR Chaudhuri were involved in data acquisition and L Bergmann was involved in the statistical analysis. All authors agree to be accountable for all aspects of the work, ensuring the accuracy and integrity of the publication.

\section{Financial \& competing interests disclosure}

This study was supported by AbbVie Inc., North Chicago, IL, USA. AbbVie participated in the study design, research, data collection, analysis and interpretation of data, writing, reviewing and approving the manuscript for publication. W Poewe was a study investigator and has received compensation from AbbVie, Astra Zeneca, Teva, Novartis, BIAL, Biogen, Britannia, Neuroderm, UCB, Orion Pharma, Takeda, Roche, Zambon and Merz Pharmaceuticals (for consultancy and lecture fees in relation to clinical drug development programs for Parkinson's disease) outside the submitted work. He has also received royalties from Thieme, Wiley-Blackwell and Oxford University Press. KR Chaudhuri was a study investigator and has received honorarium from UCB, AbbVie, Britannia, Mundipharma, Boehringer Ingelheim and GSK Pharmaceuticals for lecturing at symposia. He has acted as a consultant for UCB, AbbVie, Britannia, Neuronova and Mundipharma. KRC acknowledges independent research presented in this paper partly funded by the National Institute for Health Research (NIHR) Biomedical Research Centre at South London and Maudsley NHS Foundation Trust and King's College London. The views expressed are those of the author(s) and not necessarily those of the NHS, the NIHR or the Department of Health. He has also received research funding from Parkinson's UK, PDNMG, EU Horizon 2020 and IMI initiative, as well as educational grants from UCB, Britannia, AbbVie, GSK Pharmaceuticals, Boehringer Ingelheim and Neuronova. In addition, he has received royalties from Oxford University Press and holds intellectual property rights for the Kings Parkinson's Pain Scale and Parkinson's Disease Sleep Scale 2. L Bergmann is an employee of AbbVie and owns stock or stock options. A Antonini 
was a study investigator and has received compensation for consultancy and speaker-related activities from UCB, Boston Scientific, Boehringer Ingelheim, AbbVie and Zambon. He has received research support from Mundipharma. The authors have no other relevant affiliations or financial involvement with any organization or entity with a financial interest in or financial conflict with the subject matter or materials discussed in the manuscript apart from those disclosed.

Medical writing support, funded by AbbVie, was provided by KM Cameron of JB Ashtin, who developed the first draft based on an author-approved outline and assisted in implementing author revisions.

\section{Data sharing statement}

AbbVie is committed to responsible data sharing regarding the clinical trials we sponsor. Access is provided to anonymized, patient and trial-level data (analysis datasets), as well as other information (e.g., protocols and Clinical Study Reports) from AbbViesponsored Phase II-IV global interventional clinical trials conducted in patients (completed as of May 2004, for products and indications approved in either the USA or the European Union), as long as the trials are not part of an ongoing or planned regulatory submission). This includes requests for clinical trial data for unlicensed products and indications.

Access to this clinical trial data can be requested by any qualified researchers who engage in rigorous, independent scientific research, and will be provided following review and approval of a research proposal and Statistical Analysis Plan (SAP) and execution of a Data Sharing Agreement (DSA). Data requests can be submitted at any time and the data will be accessible for 12 months, with possible extensions considered. For more information on the process, or to submit a request, visit the following link: https://www.abbvie.com/our-science/clinical-trials/clinical-trials-data-and-information-sharing/data-and-information -sharing-with-qualified-researchers.html

\section{References}

Papers of special note have been highlighted as: $\bullet$ of interest; $\bullet \bullet$ of considerable interest

1. Mouradian MM, Heuser IJ, Baronti F, Fabbrini G, Juncos JL, Chase TN. Pathogenesis of dyskinesias in Parkinson's disease. Ann. Neurol. 25(5), 523-526 (1989).

2. Obeso JA, Olanow CW, Nutt JG. Levodopa motor complications in Parkinson's disease. Trends Neurosci. 23(10 Suppl.), S2-S7 (2000).

3. Antonini A, Moro E, Godeiro C, Reichmann H. Medical and surgical management of advanced Parkinson's disease. Mov. Disord. 33(6), 900-908 (2018).

4. Chaudhuri KR, Poewe W, Brooks DJ. Motor and nonmotor complications of levodopa: phenomenology, risk factors, and imaging features. Mov. Disord. 33(6), 909-919 (2018) (In press).

5. Nutt JG. Pharmacokinetics and pharmacodynamics of levodopa. Mov. Disord. 23(Suppl. 3), S580-S584 (2008).

6. Antonini A, Chaudhuri KR, Martinez-Martin P, Odin P. Oral and infusion levodopa-based strategies for managing motor complications in patients with Parkinson's disease. CNS Drugs 24(2), 119-129 (2010).

7. Fernandez HH, Standaert DG, Hauser RA et al. Levodopa-carbidopa intestinal gel in advanced Parkinson's disease: final 12-month, open-label results. Mov. Disord. 30(4), 500-509 (2015).

- Pivotal levodopa-carbidopa intestinal gel (LCIG) study.

8. Olanow CW, Kieburtz K, Odin P et al. Continuous intrajejunal infusion of levodopa-carbidopa intestinal gel for patients with advanced Parkinson's disease: a randomised, controlled, double-blind, double-dummy study. Lancet Neurol. 13(2), 141-149 (2014).

\section{- Pivotal LCIG study.}

9. Slevin JT, Fernandez HH, Zadikoff C et al. Long-term safety and maintenance of efficacy of levodopa-carbidopa intestinal gel: an open-label extension of the double-blind pivotal study in advanced Parkinson's disease patients. J. Parkinsons Dis. 5(1), 165-174 (2015).

\section{- $\quad$ Pivotal LCIG study.}

10. Standaert DG, Rodriguez RL, Slevin JT et al. Effect of levodopa-carbidopa intestinal gel on non-motor symptoms in patients with advanced Parkinson's disease. Mov. Disord. Clin. Pract. 4(6), 829-837 (2017).

11. Antonini A, Poewe W, Chaudhuri KR et al. Levodopa-carbidopa intestinal gel in advanced Parkinson's: final results of the GLORIA registry. Parkinsonism Relat. Disord. 45, 13-20 (2017).

\section{-. Final results from the GLORIA registry.}

12. Antonini A, Yegin A, Preda C et al. Global long-term study on motor and non-motor symptoms and safety of levodopa-carbidopa intestinal gel in routine care of advanced Parkinson's disease patients; 12-month interim outcomes. Parkinsonism Relat Disord. 21(3), 231-235 (2015).

- Interim analysis of the GLORIA registry.

13. Lv Q, Zhang B. Application of the concept of continuous dopaminergic stimulation for the management of Parkinson's disease. Neurosci. Bull. 29(5), 661-669 (2013). 
14. Wright BA, Waters $\mathrm{CH}$. Continuous dopaminergic delivery to minimize motor complications in Parkinson's disease. Expert Rev. Neurother. 13(6), 719-729 (2013).

15. Nyholm D, Odin P, Johansson A et al. Pharmacokinetics of levodopa, carbidopa, and 3-O-methyldopa following 16-hour jejunal infusion of levodopa-carbidopa intestinal gel in advanced Parkinson's disease patients. AAPS J. 15(2), 316-323 (2013).

16. Olanow CW, Obeso JA, Stocchi F. Continuous dopamine-receptor treatment of Parkinson's disease: scientific rationale and clinical implications. Lancet Neurol. 5(8), 677-687 (2006).

17. Fernandez HH, Boyd JT, Fung VSC et al. Long-term safety and efficacy of levodopa-carbidopa intestinal gel in advanced Parkinson's disease. Mov. Disord. 33(6), 928-936 (2018).

18. Timpka J, Fox T, Fox K et al. Improvement of dyskinesias with L-dopa infusion in advanced Parkinson's disease. Acta Neurol. Scand. 133(6), 451-458 (2016).

19. Antonini A, Fung VS, Boyd JT et al. Effect of levodopa-carbidopa intestinal gel on dyskinesia in advanced Parkinson's disease patients. Mov. Disord. 31(4), 530-537 (2016).

20. Politis M, Sauerbier A, Loane C et al. Sustained striatal dopamine levels following intestinal levodopa infusions in Parkinson's disease patients. Mov. Disord. 32(2), 235-240 (2017). 